\title{
Das Sorgerecht in Frankreich
}

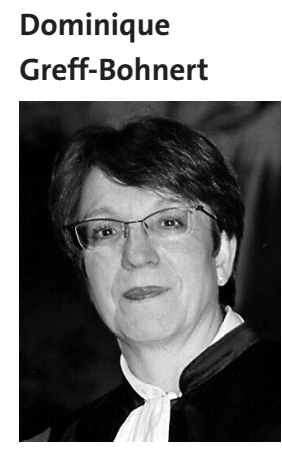

Vorsitzende Richterin am OLG Paris
Sehr geehrte Kolleginnen und Kollegen, meine Damen und Herren,

es ist mir eine große Ehre aber auch eine wahre Freude, heute im erlesenen Kreise des Deutschen Juristenbundes über das gegenwärtige Familienrecht in Frankreich, insbesondere über die Entwicklungen der elterlichen Sorge zu referieren. Diese Thematik hat übrigens einen persönlichen Hintergrund in meiner Karriere als Richterin. Bereits in meinen frühen Jahren als Familienrichterin habe ich mich mit dem Familienrecht und dessen interessanten aber manchmal auch umstrittenen und „ärgerlichen“ Konzepten auseinandergesetzt. Diese interne, nationale Auseinandersetzung wurde zudem durch deutsch-französische Erfahrungen ergänzt, besonders zu der Zeit, in der ich in den achtziger Jahren am Landgericht in Straßburg als Familienrichterin tätig war. Damals hatte ich enge Kontakte zu einer Richterin am Amtsgericht Offenburg auf Grund gemeinsamer, grenzüberschreitender Verfahren zwischen Elsass und Baden-Württemberg geknüpft und lange Jahre gepflegt. In unseren Gesprächen war es damals immer schwierig für mich, den Ausdruck „autorité parentale“/,elterliche Sorge“ in die deutsche Sprache zu übersetzen: dabei kam immer wieder „ärgerliche“ statt „elterliche“ Sorge hervor. Dieser Sprachenfehler mag wohl witzig klingen, er deutet jedoch auch auf eine akute und tagtägliche Wirklichkeit der Familien, in Frankreich und in Deutschland über Jahrzehnte hinweg, hin.

Die Zahl der Eheschließungen in Frankreich ist drastisch gesunken: im Jahr 2000 waren es noch 305.234, elf Jahre später (2011) waren es nur noch 241.000 Eheschließungen. Dieser Entwicklung entspricht die Steigerung der sogenannten „PACS“ (= Pactes Civils de Solidarité), der Solidargemeinschaftsverträge zwischen zwei Personen, die durch ein Gesetz vom 15. November 1999 in das französische Recht eingeführt wurden. Im Jahr 2000 wurden sogleich 16.859 PACS abgeschlossen, elf Jahre später (2011) waren es bereits 196.415. Parallel zu dieser Entwicklung verlief ebenfalls die Anzahl der eheähnlichen Partnerschaften, die von 322.093 im Jahre 2000 auf 448.069 im Jahr 2011 gestiegen ist. Die Zahl der Scheidungsfälle ist in Frankreich eher stabil geblieben und kennt sogar einen Rückgang: 2007 wurden 134.477 Scheidungsentscheidungen gefällt, 2013 waren es 124.411, wobei die allgemeine Arbeitslast der Familienrichter im Jahr 2013 bei 356.232 Verfahren gelegen hat. Auch diese Zahl ist im Vergleich mit dem Jahr 2010 im Rückgang, mit noch 385.883 Verfahren. Ein Zeichen der Wirtschaftskrise ...?

Mittlerweilen wurden die klassischen Verfahren zwischen heterosexuellen Partnern durch eine neue
Generation von Verfahren zwischen homosexuellen Partnern ergänzt. Im Jahr 2000 haben 5.412 homosexuelle Paare einen PACS abgeschlossen, zehn Jahre später (2010) sind es 9.143. Die jüngste Einführung der sogenannten „Ehe für Alle“ („mariage pour tous“), der homosexuellen Eheschließung hat bislang nur am Rande zu gerichtlichen Streitigkeiten geführt. Im Gegensatz dazu gibt es schon zahlreiche Verfahren zu Abstammungsfeststellung bei homosexuellen Partnerschaften.

Bevor wir miteinander in die Gegenwart des Familienrechts in Frankreich einsteigen, möchte ich zuvor noch deren geschichtlichen Hintergrund kurz erwähnen. Die Entwicklungen des Scheidungsrechts standen immer schon in engem Zusammenhang mit jenen des Abstammungsrechts. Bis 1970 war lediglich die Rede von väterlicher Verantwortung. Ein Kind sollte nur im Rahmen einer Ehe zur Welt gebracht werden, seine Rechte konnten nur durch die Ehe der Eltern zur Geltung kommen, der Vater war einziger Urheber der sogenannten „väterlichen Verantwortung“. Das Gesetz erkannte dem unehelichen Kind keine Rechte an. Erst seit einem Gesetz vom 4. Juni 1970 ist in Frankreich der Begriff „elterliche Sorge“ aufgekommen. Bei Scheidung wird meistens die elterliche Sorge (manchmal ärgerliche Sorge ...) der Mutter zugeschrieben. Bei unehelichen Kindern wurde das Sorgerechte der verlassenen Mutter, deren Rechtstand nicht besonders beneidenswert war, anerkannt.

Die Einführung der einvernehmlichen Scheidung durch ein Gesetz vom 11. Juli 1975 hat zu einer weiteren Entwicklung des Sorgerechts geführt. Insgesamt wurde das französische Familienrecht durch allein erziehende Elternteile tiefgreifend geprägt und geändert. Das jüngste Gesetz zum Scheidungsrecht - ein Gesetz vom 26. Mai 2004 - hat die Sittenentwicklung im Familienbereich weitgehend begleitet und das Sorgerecht neu gestaltet. Auch der Familienrichter ist von „juge aux affaires matrimoniales“/JAM (= Richter für eheliche Angelegenheiten) zum „juge aux affaires familiales/JAF (= Richter für familienrechtlichen Angelegenheiten) umbenannt worden. Neuere Gesetzesvorhaben sind sogar bereit, die familienrechtlichen Verfahren außergerichtlich vor dem Standesbeamten oder dem Notar abwickeln zu lassen. Ein Gesetz vom 22. Dezember 2010 sah bereits vor, dass bei Scheidungen die Suche nach einvernehmlichen Lösungen gefördert werden soll.

Auch das Kindesrecht hat eine ähnliche, parallele Entwicklung erlebt, den Kindern wurden mehr Rechte, insbesondere während des Scheidungsverfahrens der Eltern, anerkannt. Kinder werden nunmehr ge- 
richtlich angehört. Internationale Rechtsinstrumente haben das französische Familienrecht eindeutig zu wesentlichen Fortschritten geführt: unter den wichtigsten Übereinkommen möchte ich an dieser Stelle die europäische Menschenrechtskonvention, die internationale Kinderrechtskonvention (Art. 12), die europäische Kinderrechtskonvention vom 1. August 2007 und die europäische Grundrechte-Charta vom 7. Dezember 2000 zitieren. Die Cour de cassation, unser „BGH“, hat in einem Grundsatzurteil vom 18. Mai 2005 dem Kindeswohl bei Sorgerechtsstreitigkeiten einen vorrangigen Stellungswert zuerkannt.

Gefördert durch die Entwicklung der Familie ist das Konzept der elterlichen Sorge erst zum Vorschein gekommen und hat aber auch eine rasche Verwandlung erfahren.

\section{Das Sorgerecht als Konzept}

\section{Begriffsbestimmung}

Das elterliche Sorgerecht ist durch ein Gesetz vom 4. März 2002 entstanden. Dieses Gesetz hat das Abstammungsrecht tiefgreifend modernisiert und hat zum ersten Mal eine eindeutige Definition des Sorgerechts eingeführt und es als eine, allein auf das Kindeswohl zielende Gesamtheit von Rechten und Pflichten bezeichnet. Das Sorgerecht steht den Eltern, also dem Vater und der Mutter bis zur Volljährigkeit oder Emanzipation des Kindes zu. Es dient dem Schutz seiner Sicherheit, seiner Gesundheit und seiner Sittlichkeit und gewährleistet seine Erziehung und persönlichen Entfaltung. Die Eltern sind gefordert, das Kind je nach Alter und Reife in ihre Entscheidungen mit einzuschließen.

\section{Gemeinsames Sorgerecht als Regelfall}

Unter der Voraussetzung, dass die Abstammung des Kindes den beiden Elternteilen gegenüber festgestellt ist, wird das Sorgerecht beiden Eltern gleichermaßen als gemeinsam auszuübendes Recht anerkannt. Dies betrifft auch ein Elternteil, das selbst noch minderjährig ist. Das Sorgerecht ist unabdingbar, beiden Eltern auferlegt und dauert von der Geburt bis zur Volljährigkeit oder Emanzipation an.

Es gibt keinen Sorgerechtsverlust bei Trennung oder Scheidung, alleine der Familienrichter hat die Befugnis, die elterliche Sorge zu entziehen.

Die Eltern sind verpflichtet, das Kind zu schützen, zu ernähren und zu erziehen. Das Konzept des Kindeswohls ist insofern zum Eckpfeiler des Sorge- und Kindesrechts geworden.

\section{Alleiniges Sorgerecht als Ausnahme}

Alleiniges Sorgerecht besteht dann, wenn das Kind nur über ein einziges Abstammungsverhältnis verfügt oder wenn der zweite Elternteil verstorben ist oder wenn das Sorgerecht einem der beiden Eltern gerichtlich entzogen worden ist. Das Sorgerecht kann einem Dritten übertragen werden: es besteht hierzu eine ausführliche Rechtsprechung für nicht mütterliche homosexuelle Lebenspartnerinnen (die sich aber trotzdem als „Mütter“ betrachten), wenn das Kind nach einer, in Belgien ausgeübten medizinisch unterstützten Fortpflanzung zur Welt gekommen ist.
Bevor der Richter ein alleiniges Sorgerecht zuspricht, muss er die Fähigkeit beider Elternteile zur Pflichtenerfüllung prüfen, eventuelle Sachverständigengutachten einholen, das Kind anhören, sein Alter und die Ergebnisse eventueller Gutachten zum sozialen Umfeld des Kindes beachten. Seit einem Gesetz vom 9. Juli 2010 zum Schutz der Frauen muss der Richter auch eventuelle Drohungen oder physische sowie psychische Gewalttaten, die durch einen Elternteil auf den anderen ausgeübt worden sind, in Betracht ziehen und berücksichtigen. Diese Berücksichtigung findet auch dann statt, wenn das Kind nicht selber Opfer von Gewalttaten ist.

\section{Der Verfall oder die Entziehung des Sorgerechts}

Das Sorgerecht kann einem Elternteil entzogen werden. Dies geschieht insbesondere wenn der Vater oder die Mutter auf Grund einer gesundheitlichen Behinderung, seiner Abwesenheit oder irgendeiner Unfähigkeit nicht mehr in der Lage ist, die elterliche Sorge seinem Kind gegenüber auszuüben. Das Sorgerecht wird dann durch den anderen Elternteil alleine ausgeübt. Sollten beide Eltern das Sorgerecht verloren haben, wird das Kind unter Vormundschaft gestellt.

Die Entziehung des Sorgerechts kann in einem strafrechtlichen Urteil wegen Verbrechens oder Vergehens zum Nachteil des Kindes erlassen werden. Sie kann aber auch außerhalb eines strafrechtlichen Rahmens entschieden werden, wenn Misshandlungen, massiver Alkohol- oder Drogenkonsum, ungebührlicher Lebenswandel, strafrechtlich relevantes Verhalten, Unterlassung der Unterhaltszahlungen oder der Erziehung des Kindes den Eltern vorgeworfen und nachgewiesen werden können. Diese Voraussetzungen sind in Art. 378-1 des Code civil vorgesehen und müssen zur Gefährdung der Sicherheit, der Gesundheit oder der Sittlichkeit des Kindes geführt haben. Solche Situationen werden meistens dem Jugendrichter berichtet und führen zu dessen gerichtlichen Anrufung. Die Entziehung kann gegebenenfalls auch nur teilweise ausgesprochen werden.

\section{Die Ausübungsbestimmungen des Sorgerechts}

Durch ein Gesetz vom 4. März 2002 ist der Zusammenhang zwischen Verwandtschaft und Ehe abgeschafft worden. Daher ist auch die rechtliche Unterscheidung zwischen ehelichen und nichtehelichen Kindern weggefallen. Die Wahrnehmung der elterlichen Verantwortung ist nunmehr selbstständig und die Ausübung des Sorgerechts betrifft alle Kinder ungeachtet ihrer Abstammung oder Rechtsstellung den Eltern gegenüber.

\section{A. Die gemeinsame Elternschaft}

\section{Gemeinsames Sorgerecht als Regelfall}

Das gemeinsame Sorgerecht gilt als Regelfall, auch bei nicht miteinander verheirateten Eltern. Die einzige Ausnahme betrifft Kinder aus nichtehelichen Partnerschaften, die spätestens ein Jahr nach der Geburt anerkannt werden müssen: das Sorgerecht wird dann automatisch gemeinsam ausgeübt. Dasselbe gilt ebenfalls für adoptierte Kinder. Wenn ein Ehegatte das leibliche Kind seines Gatten adoptiert, kommt das Sorgerecht laut Art. 365 des Code 
civil allein dem leiblichen Elternteil zu, es sei denn, beide Ehegatten geben eine gemeinsame Erklärung bei der Geschäftsstelle des Landgerichts zur gemeinsamen Ausübung des Sorgerechts ab. Diese Vorschrift betrifft lediglich verheiratete Eltern und ist bei nichtehelichen Partnerschaften ausgeschlossen. Die Adoption bleibt daher der Partnerin der Mutter verweigert. Seit dem Gesetz zur sogenannten „Ehe für Alle“ von 2013 stellt sich das gleiche Problem nun für homosexuelle Ehen. Eine Grundsatzentscheidung der Cour de cassation ist zwar noch nicht gefällt worden, man vermutet jedoch, dass der oberste Gerichtshof einer Adoption nicht zustimmen würde, obgleich die Familienrichter immer öfter solche Adoptionen für gültig erklären.

Vater und Mutter haben Rechte und Pflichten den Kindern gegenüber. Jede elterliche Entscheidung bedarf der Einwilligung beider Elternteile. Die Ausübung des Sorgerechts verläuft gemeinsam, nicht konkurrierend. Art. 372-2 des Code civil erlaubt jedoch beiden Eltern, gewöhnliche Handlungen des täglichen Lebens alleine und eigenständig zu verrichten. Anders verhält es sich bei nicht gewöhnlichen Handlungen, also bei außergewöhnlichen oder komplexen Verrichtungen, die eine Einwilligung beider Elternteile voraussetzen. Diese Verpflichtung ist auch Dritten gegenüber wirksam.

Gutgläubige Dritte dürfen davon ausgehen, dass jeder Elternteil seine Zustimmung zur Handlung dem anderen bereits erteilt hat. Das Gesetz gibt keine Begriffsbestimmung zur ,gewöhnlichen Handlung “ und hat auch keine Beispiele aufgelistet. Man kann aber davon ausgehen, dass es keine einfache, normale Handlung ist, wenn sie mit der Vergangenheit bricht oder wenn sie die Zukunft des Kindes eindeutig belasten wird.

An dieser Stelle kann man folgende Beispiele einblenden:

- Im Schulbereich:

- Die Ersteinschreibung des Kindes an einer Schule wird nicht als „einfache Handlung“ bewehrtet; seine Wiedereinschreibung entgegen wird als solche betrachtet.

- Das gleiche gilt bei der Wahl einer Privatschule oder eines europäischen Lehrganges an einem Gymnasium.

- Anderseits wiederum ist die Einschreibung in einer Schulkantine, in einer Abendnachhilfe oder an einem Schulausflug eine „einfache Handlung“, die jedem einzelnen Elternteil alleine zusteht.

- Im Gesundheitsbereich:

- Alltägliche Pflegeleistungen, sogar Zwangsimpfungen gelten als „einfache Handlungen“.

- Schwere medizinische Behandlungen sowie andauernde Aufenthalte in einem Krankenhaus oder kieferorthopädische Behandlungen werden selbstverständlich nicht als „einfache Handlungen“ eingestuft und bedürfen einer gemeinsamen elterlichen Einwilligung.

- Im Bereich des Personenstandes:

- Die Wahl eines Vornamens oder eines Namens und der Verzicht auf eine Staatsangehörigkeit sind keine „einfachen Handlungen“.

- Anders verhält es sich bei der Eintragung eines Kindes im Pass der Eltern, die eindeutig als „einfache Handlung“ betrachtet wird.
- Im Bereich der Religion:

- Beschneidung, Taufe oder Glaubensbekenntnis sind keine „einfachen Handlungen“.

Sind sich beide Eltern nicht einig, dann:

a) Kann eine Mediation (Schlichtung) als Alternativverfahren eingeschaltet werden (zurzeit nur versuchsweise). Der Code civil setzt dem Richter zur Vorgabe, den Parteien möglichst im Wege eines Ausgleichs zur Versöhnung zu verhelfen. In diesem Sinne ermächtigt Art. 373-2-10 den Richter, eine Mediation zwischen den Eltern anzuordnen. Bevor diese

Lösung jedoch zu einer verbindlichen gesetzlichen Vorschrift wird, wird sie einstweilen in manchen Landgerichten bis Ende 2014 ausprobiert.

b) Hat der Familienrichter als zuständige Gerichtsbarkeit zu entscheiden. Das Gesetz vom 4. März 2002 legt ihm auf, die Streitigkeit mit dem Ziel auf das alleinige Kindeswohl beizulegen. In der Praxis kommt es allerdings selten zur Anrufung des Familiengerichts, wenn beide Eltern miteinander leben.

\section{Alleiniges Sorgerecht als Ausnahmefall}

Dem Elternteil, dem das Sorgerecht nicht zugesprochen worden wurde, stehen jedoch manche Rechte weiterhin zu. Sie bilden ein Gleichgewicht zu seinen Verpflichtungen dem Kind gegenüber. Er verfügt weiter über das Recht, einer Eheschließung des Kindes, seiner Adoption oder seiner Emanzipation zuzustimmen. Er darf seine Beziehungen zum Kind weiterhin im Wege des Umgangsrechts, das ihm nur aus schwerwiegenden Gründen entzogen werden kann, fortsetzen und pflegen. Er darf ferner mit dem Kind schriftlich verkehren, was heutzutage mehr und mehr über Internet oder Skype stattfindet.

Sind sich die Eltern uneinig, dann:

- Kann eine Mediation - wie zuvor erwähnt - angeordnet werden;

- Muss daran erinnert werden, dass der Elternteil, dem das Sorgerecht nicht zugesprochen worden wurde, weiterhin für den Unterhalt und die Erziehung seines Kindes verantwortlich bleibt. Er muss durch den anderen Elternteil über wichtige Entscheidungen für das Leben des Kindes unterrichtet werden. Die häufigsten Anrufungen des Familiengerichts finden im Bereich der Wahl des Aufenthaltsortes des Kindes und des Beitrags zu seinem Unterhalt und seiner Erziehung statt.

\section{B. Die Ausübung des Sorgerechts bei getrennten Eltern}

\section{Die Ermessungskriterien}

Die Schwierigkeiten erscheinen bekanntlich bei der Trennung der Eltern. Der Familienrichter ist dann alleine zuständig, um sorgerechtsrelevante Konflikte beizulegen.

Wie bereits erwähnt, hat die Trennung der Eltern keinerlei Einfluss auf das Sorgerecht. Sie ist weder ein Grund zur Entziehung des gemeinsamen Sorgerechts noch zur Bestimmung des alleinigen Sorgerechts zu Gunsten eines der beiden Elternteile. Nach ständiger Rechtsprechung ist es kein Grund, die gemeinsa- 
me elterliche Sorge aufzulösen und einem der beiden Elternteile alleine zu übertragen, wenn:

- Ein akuter Konflikt zwischen den Eltern besteht;

- Die Eltern in weiter Entfernung leben;

- Vater oder Mutter der Erziehung des Kindes nicht gerecht werden oder ihr Umgangsrecht nicht ausüben;

- Ein strafrechtliches Urteil wegen einer Straftat (Diebstahl, z.B.) erlassen worden ist.

Sollte der Familienrichter vom gemeinsamen Sorgerecht abweichen wollen, muss er eine gesonderte, auf das Kindeswohl gestützte Begründung aufbringen.

In der Praxis ist die alleinige elterliche Sorge wirklich eine Ausnahme und wird nur in Extremfällen ausgesprochen. Dies ist dann der Fall, wenn ein Elternteil eine Bedrohung für das Kindeswohl darstellt oder wenn das Kindeswohl selbst eine solche Lösung erfordert. Zum Beispiel, wenn ein Elternteil seine Beziehung zum Kind gänzlich vernachlässigt : bei völliger Unterlassung des Umgangsrechts oder der Unterhaltszahlungen, bei grober Gleichgültigkeit gegenüber der Gesundheit oder der Schulung des Kindes, bei Abbruch jeglicher Beziehung zum Kind, bei heimlicher Beschneidung des Kindes unter Missachtung der ausdrücklichen Verweigerung des anderen Elternteils oder darüber hinaus bei ernsthafter Drohung einer Kindesentführung ins Ausland. Diese Ermessungskriterien klingen in Wirklichkeit wie eigentliche Sanktionsgründe.

Zur Festlegung des Umgangsrechts oder des Kindeswohnsitzes gibt das Gesetz dem zuständigen Richter unter Art. 373-5-11 des Code civil folgende Ermessungskriterien:

- Die Gewohnheiten der Eltern und ihre bereits getroffenen Vereinbarungen.

- Die durch das minderjährige Kind geäußerten Neigungen.

- Die Schlussfolgerungen eines eventuellen Gutachtens je nach Kindesalter.

- Die Ergebnisse eines Gutachtens über das soziale Umfeld des Kindes.

- Die Feststellung bei einem Elternteil von körperlichen oder psychischen Drohungen oder Gewalttaten, die durch den anderen Elternteil ausgeübt worden sind.

An dieser Stelle möchte ich folgende Bemerkungen machen:

\section{a) Zur Anhörung des Kindes}

Mit Blick auf die Rechtsprechung des Europäischen Gerichtshofes für Menschenrechte und auf die ratifizierten internationalen Abkommen hat der französische Gesetzgeber die Anhörung eines urteilsfähigen Kindes bereits in einem Gesetz vom 8. Januar 1993 erlaubt. Diese Anhörung ist $2007 \mathrm{zu}$ einem eigenständigen Recht eingestuft worden und muss bei vorherigem Antrag des Kindes zwingend angeordnet werden. Laut Art. 388-1 des Code civil wird ein urteilsfähiges Kind entweder unmittelbar durch den Richter selbst oder bei Bedarf durch eine vom Richter beauftragte Person angehört. Die Anhörung, die dem Kind nicht die Eigenschaft der Prozesspartei verleiht, kann in Anwesenheit eines Rechtsbeistands oder einer frei gewählten Person stattfinden.

In der Praxis wird das Kind zu Beginn des Verfahrens durch den Rechtsbeistand oder durch die Gerichtsgeschäftsstelle über dieses besondere Recht belehrt. In den meisten Fällen und besonders am OLG Paris werden die Kinder durch den Richter persönlich angehört. Die Anhörung findet im Arbeitszimmer des Richters in Anwesenheit des Rechtsbeistandes statt. Der Inhalt der Anhörung wird mit Einwilligung des Kindes nur insofern zu Protokoll gesetzt, wenn es gilt, die Eltern darüber zu

informieren. Eine Ablehnung der Anhörung kann nur auf Grund der Urteilsunfähigkeit des Kindes erfolgen. Diese Fähigkeit beginnt laut Rechtsprechung im Alter von etwa sieben oder acht Jahren. Die Anhörung kann während des Verfahrens zu jeder Zeit, sogar nach Abschluss der Hauptverhandlung vorgenommen werden. Der Familienrichter ist jedoch nie durch die vom Kind geäußerte Meinung gebunden.

\section{b) Zu den anderen richterlichen Ermessungskriterien}

Es ist mir hier leider nicht möglich, sämtliche Kriterien eingehend zu erörtern. Ich werde daher nur kurze Randbemerkungen zu den gesetzlichen Kriterien vortragen.

Als erster Anhaltspunkt wird der Richter die durch die Eltern bereits getroffenen Vereinbarungen in Betracht ziehen. Anschließend prüft er ihre Fähigkeit, den elterlichen Pflichten gerecht zu werden und die Rechten des jeweiligen anderen Elternteils zu achten und einzuhalten. Seit einer Grundsatzentscheidung der Cour de cassation vom 4. Juli 2006 wird diesem Ermessungskriterium eine besonders große Rolle in der Urteilsfindung zugemessen. Hierzu zwei Beispiele: der heimliche Umzug eines Elternteils oder noch die systematische Herabsetzung des anderen Elternteils vor dem Kind. Auch die jüngste Gesetzgebung vom 9. Juli 2010 zum Schutz der Frauen als Opfer häuslicher Gewalt wird als neues Ermessungskriterium verwendet, zumal sie eine Prüfung der gegenseitigen Fähigkeit zur Achtung der Rechte ermöglicht.

Bei Trennung der Eltern gehen die Streitigkeiten bekanntlich um den Wohnsitz des Kindes, das Umgangsrecht und den finanziellen Unterhaltsbeitrag.

\section{Die Festlegung des Wohnsitzes}

Dies ist meistens die Kernstreitigkeit getrennter Eltern. Ein Gesetz vom 4. März 2002 hat die vorherige, von der Cour de cassation in einem Grundsatzurteil vom 2. Mai 1984 gebilligte Praxis der paritätischen Betreuung gesetzlich verankert. Laut Art. 373-2-9 Abs.1 des Code civil kann der Wohnsitz des Kindes nunmehr abwechselnd entweder am Wohnort beider Eltern oder am Wohnort eines einzelnen Elternteils festgelegt werden.

\section{a) Der abwechselnde Wohnsitz}

Erlauben Sie mir, dieses Konzept an dieser Stelle etwas zu vertiefen, da die paritätische Betreuung oft im Vordergrund der elterlichen Streitigkeiten liegt. Sie versinnbildlicht auch eine schwerwiegende Änderung in unserer modernen Gesellschaft, in der beide Eltern meistens berufstätig sind und nur verkürzt Zeit gemeinsam miteinander verbringen. Ihr persönliches Leben wird oft während der Minderjährigkeit des Kindes mehrfach in der Form der sogenannten „Patchwork-Familie“ umgestaltet. 
Der abwechselnde Wohnsitz kann entweder von den Eltern gewählt oder durch den Familienrichter angeordnet werden. Eine Probezeit von 6 Monaten kann wahlweise verhängt werden. Der Richter hat die Befugnis, den abwechselnden Wohnsitz zu verweigern, wenn er dem Kindeswohl zuwiderlaufen würde.

Der abwechselnde Wohnsitz gilt seit 2002 als eine gesetzliche Alternative, die sowohl dem Richter als auch den Eltern zusteht. Sie bedarf keiner verbindlichen Einwilligung beider Elternteile, eine Streitigkeit der Eltern zu dieser Frage bildet auch keinen rechtlichen Grund für den Richter, den abwechselnden Wohnsitz auszuschließen. Die richterliche Würdigung geschieht stets „in concreto " und ist ausschließlich auf das Kindeswohl gezielt und gestützt. Das OLG Paris - und mein Senat insbesondere - sehen einem abwechselnden Wohnsitz für sehr junge Kinder besonders kritisch entgegen. So wurden z.B. die extrem schlechten Verhältnisse zwischen beiden Elternteile wegen regelmäßiger häuslicher Gewalt als Ablehnungsgrund eingestuft. Dies wird übrigens auch dann der Fall sein, wenn es darum geht, den betroffenen Kindern einen sicheren und geborgenen Lebensrahmen zu gewährleisten, obgleich beide Elternteile ihren Wohnsitz in derselben Gemeinde haben.

Auch im Nachhinein kann der abwechselnde Wohnsitz auf Grund des Kindeswohls geändert oder abgeschafft werden. Dies kann z.B. dann eintreten, wenn die Schulergebnisse des Kindes plötzlich sinken, wenn es durch ständiges Wechseln des Wohnortes verunsichert wird oder auch wenn das Kind selber dem Richter einen entsprechenden Antrag stellt. Diese Kriterien sind paradoxerweise aber zugleich auch gute Gründe, um einen abwechselnden Wohnsitz anzuordnen.

Die Festlegung des Zeittaktes der Abwechslung ist dem alleinigen Ermessen des Richters überlassen. Meistens verbleibt das Kind eine Woche bei einem Elternteil, die darauf folgende Woche dann beim anderen Elternteil, der Wechsel findet gewöhnlich freitags statt. Die Schulferienzeit wird halbiert, wobei gerechte Zeitaufteilung nicht unbedingt egalitäre Aufteilung bedeutet.

Ein abwechselnder Wohnsitz hat auch materielle und finanzielle Folgen im Alltag. Meistens - wenn nicht anders vereinbart werden Kindergeld und weitere Familienzulagen gleichwohl wie sämtliche Ausgaben zum Unterhalt des Kindes zwischen den Eltern zur Hälfte geteilt. Der jeweilige Elternteil haftet für sein Kind während dessen Residenzzeit bei ihm. Diese Haftung bedarf jedoch noch einer erwarteten Bestätigung der Cour de cassation.

In zehn Jahren ist der abwechselnde Wohnsitz von 8,8 Prozent auf 14,9 Prozent der Fälle gestiegen. Davon sind in der Praxis 95 Prozent der Fälle auf eine Vereinbarung der Eltern zum abwechselnden Wohnsitz gestützt. Bei Uneinigkeit der Eltern sinkt die Quote auf 50 Prozent.

\section{b) Der feste Wohnsitz des Kindes bei einem der Eltern}

In ihrer Rechtsprechung erinnert die Cour de cassation systematisch an das Kindeswohl als einziger Maßstab der richterlichen Bewertung und Würdigung. Hierzu können folgende Kriterien dienen:

- Die Stabilität der Lebensbedingungen des Kindes.

- Seine Sicherheit.

- Die Verfügbarkeit der Eltern ihrem Kind gegenüber.
- Die Achtung der Rechte des anderen Elternteils.

- Der Wille des Kindes je nach dessen Alter.

- Die religiöse oder sexuelle Einstellung eines Elternteils.

- Die Berücksichtigung der Geschwister: ein Kind soll nur ausnahmsweise von seinen Geschwistern getrennt werden, eine Trennung bleibt dennoch möglich, wenn das Kindeswohl es erfordert.

\section{c) Der feste Wohnsitz des Kindes bei einer Drittperson}

Diese richterliche Lösung bleibt eine Ausnahme und muss wiederum verbindlich dem Kindeswohl entsprechen. Hier befindet sich der Familienrichter in Konkurrenz mit dem Jugendrichter, der bekanntlich über ausgedehnte Möglichkeiten verfügt, um das Kind einer Drittperson oder einem Kinderheim anzuvertrauen und um dessen finanziellen Unterhalt zu sichern und zu regeln.

\section{Die Aufrechterhaltung und Pflege der Beziehungen zwi- schen Eltern und Kind}

Art. 372-2 Abs. 2 des Code civil verpflichtet jeden Elternteil zur Aufrechterhaltung persönlicher Beziehungen zu seinem Kind und zur Achtung der notwendigen Beziehungen des anderen Elternteils zum Kind sowohl bei gemeinsamem als bei alleinigem Sorgerecht.

\section{a) Das Umgangsrecht}

Das Umgangsrecht wird angeordnet, wenn das Kind ausschließlich bei einem Elternteil lebt, also außerhalb des abwechselnden Wohnsitzes. Das Umgangsrecht kann nur aus schwerwiegenden Gründen durch den Richter ausgeschlossen werden. Die Rechtsprechung legt diese Ausnahme eher restriktiv aus: als schwerwiegende Gründe gelten eine Verurteilung wegen sexuellen Missbrauchs des Kindes, Alkoholabhängigkeit eines Elternteils, Gewalttaten gegenüber dem Kind oder noch große Unregelmäßigkeiten in der Ausübung des Umgangsrechts.

\section{b) Die Einschränkungen des Umgangsrechts}

- Der Richter beschließt immer ein „Mindestumgangsrecht“. Obgleich die Ausübung des Umgangsrechts meistens einvernehmlich durch die Eltern vereinbart wird, steht es dem Richter zu, die Modalitäten der Ausübung bei Uneinigkeit festzulegen. Gewöhnlich erfasst das Umgangsrecht jede zweite Woche von Freitagabend nach der Schule bis Sonntagabend oder Montagmorgen und die Hälfte der Schulferien. Die Festlegung kann jedoch nicht dem Kindeswillen überlassen werden: die Cour de cassation schließt ungeachtet des Kindesalters jegliche Entscheidungsübertragung auf das Kind aus.

- Das Umgangsrecht kann unter Aufsicht eines Mediatoren an einem gesonderten Ort ausgeübt werden. Es handelt sich hierbei um eine neutrale Mediationsstelle, die die Sicherheit des Kindes gewährleistet. Ein Gesetz vom 5. März 2007 hat durch Sozialhilfegelder finanzierte Treffpunkte eingerichtet. Art. 379-2 des Code civil ermächtigt den Richter, ein sogenanntes mediatisiertes Umgangsrecht anzuordnen, wenn dies für das Wohl des Kindes notwendig ist oder wenn anderenfalls eine unmittelbare Übergabe des Kindes an das andere Elternteil eine Gefahr für einen der beiden darstellen 
würde. Letzteres muss allerdings zeitlich beschränkt sein und auf die Wiederaufnahme normaler Beziehungen abzielen.

- Das Umgangsrecht kann auch geografisch beschränkt werden, indem es durch ein Ausreiseverbot ergänzt wird. Solche Maßnahmen dienen selbstverständlich der Vorbeugung internationaler Kindesentführungen. Der Richter ist befugt, ein Ausreiseverbot aus Frankreich (auch Übersee), das zeitlich unbegrenzt sein kann, insofern zu verhängen, dass beide Eltern der Ausreise ihre Einwilligung geben müssen. Die Staatsanwaltschaft lässt diese richterliche Entscheidung in die Datei für Personenfahndung eintragen.

- Das Umgangsrecht kann letztendlich bei Bedarf auch reduziert oder gar völlig aufgehoben werden. Dies kann z.B. bei Erkrankung oder Behinderung des Kindes oder eines Elternteils, bei Inhaftierung oder bei Verlust des Wohnsitzes der Fall sein.

\section{Der Beitrag zum Kindesunterhalt}

Streitigkeiten über den jeweiligen Beitrag zum Kindesunterhalt tragen tagtäglich zu einer wesentlichen Arbeitslast des Familienrichters bei. Um diese Last jedoch einzudämmen, ist immer häufiger die Rede von der Einführung einer Tabelle - etwa nach deutschem Muster - für die Bemessung des Unterhaltsbeitrags. Eine Tabelle ist bereits durch das Justizministerium veröffentlicht und zu Verfügung gestellt worden. Ihre Berücksichtigung durch die Parteien ist jedoch nicht verbindlich. Der Richter kann sie auch nicht in seiner Begründung benutzen, da seine Entscheidung ausschließlich auf Einkünfte und Ausgaben der Eltern und auf Kindesbedürfnisse gestützt werden darf.

\section{Schlusswort}

Sehr geehrte Kolleginnen und Kollegen, meine Damen und Herren,

gestatten Sie mir zum Schluss noch drei kurze Bemerkungen zu machen:

- Im Bereich des Familienrechts zielt die französische Gesetzgebung auf den Konsens, die Einvernehmlichkeit ab. Das Gesetz vom 4. März 2002 verleiht den Eltern die Möglichkeit, die Sorgerechtseinzelheiten selbst auszuwählen und zu gestalten. Der Richter kann je nach Ermessen dieser Wahl Rechtskraft verleihen.

- Unser gegenwärtiges Familienrecht ermöglicht erfreulicherweise die Erziehung des Kindes durch beide Eltern auch nach deren Scheidung und fördert somit eine ausgewogene affektive Bindung zwischen Eltern und Kind.

- Das gemeinsame Sorgerecht als Regelfall ist nun im französischen Familienrecht fest verankert. Ein zurzeit noch im Parlament zur Debatte stehender Gesetzentwurf sieht sogar vor, dass der Familienrichter befugt sein wird, eine Ordnungsstrafe von bis zu 10.000 Euro zu verhängen, wenn ein Elternteil dieser Regelung bewusst Hindernisse setzt oder gar gegen eine entsprechende richterliche Entscheidung verstoßen hat. Ich bedanke mich für Ihre Aufmerksamkeit und stehe selbstverständlich für jede Frage gerne zu Verfügung.

\section{Elterliche Verantwortung in Deutschland: Verfahrensrechtliche Aspekte - Kindesanhörung und Mediation}

\section{Elterliche Verantwortung in Deutschland}

1.

Um elterliche Verantwortung kann es zum einen im Verhältnis der Eltern zum Staat gehen.

Hier heißt es in Art. 6 Abs.2 GG: „Pflege und Erziehung der Kinder sind das natürliche Recht der Eltern und die zuvörderst ihnen obliegende Pflicht. Über ihre Betätigung wacht die staatliche Gemeinschaft."

Der Freiheitsraum der elterlichen Betätigung wird im Verhältnis zum Staat wie folgt abgegrenzt ${ }^{1}$ : Diese Normen garantieren den Vorrang der Eltern, ihre Eigenständigkeit und Selbstverantwortlichkeit bei der Pflege und Erziehung der Kinder, bestellen aber zugleich die staatliche Gemeinschaft zum Wächter. Das so umgrenzte Elternrecht [...] gewährt den Eltern ein Abwehrrecht gegen staatliche Eingriffe, soweit diese nicht durch das „Wächteramt“ gedeckt sind. Die Eltern haben das Recht, die Pflege und Erziehung ihrer Kinder nach ihren eigenen Vorstellungen frei zu gestalten und genießen Vorrang vor anderen Erziehungsträgern. Dieser Grundrechtsschutz darf aber nur für ein Handeln in Anspruch genommen werden, das bei weitester Anerkennung der Selbstverantwortlichkeit der Eltern noch als Pflege und Erziehung gewertet werden kann, nicht aber für das Gegenteil: die Vernachlässigung des Kindes. [...] Wenn Eltern in dieser Weise versagen, greift das Wächteramt des Staates nach Art. 6 Abs. 2 Satz 2 GG ein; der Staat ist nicht nur berechtigt, sondern auch verpflichtet, die Pflege und Erziehung des Kindes sicherzustellen. Diese Verpflichtung

1 BVerfG, Entscheidung v. 29.7.1968 - 1 BvL 20/63, 1 BvL 31/66 -, BVerfGE 24, 119 m.w.N.

\section{Ruth Feldkemper-}

Bentrup

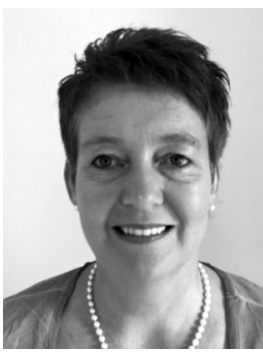

Richterin am

Oberlandesgericht Hamm, stellvertretende Vorsitzende des 11. Senats für Familiensachen 